\title{
Pediatric Hodgkin Lymphoma; Experience with Euronet Pediatric Hodgkin Lymphoma Protocol in a Developing \\ Country
}

Tariq Ghafoor ${ }^{1,2 *}$, Raheel Iftikhar ${ }^{1}$, Imtenan

Sharif', Tanveer Ashraf ${ }^{2}$, Shakeel Ahmed ${ }^{2}$, Farrah

Bashir $^{2}$

${ }^{1}$ Armed Forces Bone Marrow Transplant Centre, Pakistan ${ }^{2}$ Department of Paediatric Oncology, Pakistan

*Corresponding author: Tariq Ghafoor, Paediatric Oncologist and Bone Marrow Transplant Specialist,

Armed Forces Bone Marrow Transplant Centre, CMH

Medical Complex, Rawalpindi, Pakistan

Received: August 23, 2018; Accepted: September 24, 2018; Published: October 01, 2018

\begin{abstract}
Objective: To analyse outcome of Paediatric Hodgkin Lymphoma (HL) patients treated with Euronet Paediatric Hodgkin Lymphoma treatment protocol in a developing country.

Patients and Methods: This is a prospective study conducted at Paediatric Oncology department at Combined Military Hospital (CMH) Rawalpindi, Pakistan. All newly diagnosed cases of $\mathrm{HL}$, less than 18years registered from January 2012 onwards that completed their treatment until 30th June 2018 were included. Data regarding age, sex, staging, histopathology and outcome were analysed.

Results: Total 102 patients including 80 (78.4\%) males and $22(21.6 \%)$ females were analysed. The mean age was $7.54 \pm 2.77$ years. B symptoms were present in $58(56.9 \%)$ patients. Ten (9.8\%) patients had stage I disease, 33 (32.4\%) stage II, $41(40.2 \%)$ stage III and $18(17.6 \%)$ stage IV disease. Mixed cellularity was the commonest histological subtype seen in $74(72.5 \%)$ patients. Involved field radiotherapy was given to $17(16.7 \%)$ patients having inadequate response after 2 cycles of OEPA chemotherapy. Eight (7.8\%) patients died of treatment related complications and $10(9.8 \%)$ patients relapsed. Nine of the relapsed $(90 \%)$ cases responded to $2^{\text {nd }}$ line treatment including one requiring autologous stem cell transplant. One relapsed patient died of progressive disease. Overall Survival (OS) and Event Free Survival (EFS) was $91.2 \%$ and $82.4 \%$ respectively.
\end{abstract}

Conclusion: In Pakistan, mixed cellularity is the commonest $\mathrm{HL}$ subtype seen in young males. Long duration of disease before presentation, malnutrition, presence of B symptoms, bone marrow and lungs involvement and advanced disease is associated with decreased OS and EFS.

Keywords: Hodgkin lymphoma; Treatment outcome; Developing country; Pakistan; Euronet PHL protocol

\section{Introduction}

Treatment of Paediatric Hodgkin Lymphoma (PHL) is one of the important success stories of the previous century. Treatment protocols of PHL have evolved over many years with a consideration of reduction of acute and long-term toxicity of chemotherapy and radiotherapy. Initial treatment protocols incorporated unacceptably high radiation doses for children.

In the study GPOH-HD 95, radiotherapy was restricted to patients not in complete remission after chemotherapy [1]. Current treatment protocols are based upon a risk-based and response-adapted approach in which patients receive multi-agent chemotherapy with or without low-dose involved-field or involved-site radiation therapy [2]

Most European centres are following protocols formulated by European Network for Paediatric Hodgkin Lymphoma (EuroNetPHL) while the Paediatric Oncology Group and Children's Cancer Group have merged to form the Children's Oncology Group (COG), widely practised in North and South America. In developed countries, the 5-year survival rate of PHL increased from $81 \%$ to more than $95 \%$ between 1975 and 2010 [3]. However, survival in developing countries is still significantly lower, because of late presentation, abandonment of therapy and inadequate supportive and critical care [4-6]. Data of few published studies from Pakistan shows overall survival ranging from $76 \%$ to $84 \%$ [7]. There is no uniform protocol for treatment of PHL in Pakistan. We conducted this study using treatment as per The EuroNet-PHL-C1 protocol.

\section{Materials and Methods}

This is a prospective on-going study being carried out in the department of Paediatric Oncology at Combined Military Hospital Rawalpindi, Pakistan. The hospital ethical committee approved the study and informed consent was obtained from parents of the patients. Data collection was started in January 2012. All newly diagnosed patients with histologically confirmed primary diagnosis of classical HL, less than 18 years of age were included in the study. Patients having prior chemotherapy or radiotherapy for other malignancies, pre-treatment of HL, (except for 7-10 days steroid pre-phase of a 
Table 1: Patient characteristics.

\begin{tabular}{|c|c|c|}
\hline & Number (n) & Percentage (\%) \\
\hline Total number & 102 & 100 \\
\hline \multicolumn{3}{|l|}{ Age } \\
\hline 5-Jan & 24 & 23.5 \\
\hline$>5-10$ & 60 & 58.8 \\
\hline$>10-15$ & 18 & 17.6 \\
\hline \multicolumn{3}{|l|}{ Sex } \\
\hline Male & 80 & 78.4 \\
\hline Female & 22 & 21.6 \\
\hline \multicolumn{3}{|l|}{ Nutrition Status } \\
\hline Well-nourished & 76 & 74.5 \\
\hline Under-nourished & 26 & 25.5 \\
\hline \multirow{3}{*}{ Duration of symptoms } & \multicolumn{2}{|c|}{ Mean $8.97 \pm 8.65$ months } \\
\hline & \multicolumn{2}{|c|}{ Median 6 months } \\
\hline & \multicolumn{2}{|c|}{ (Range; 1-48 months) } \\
\hline \multicolumn{3}{|l|}{ Presentation } \\
\hline Cervical Lymphadenopathy & 85 & 83.3 \\
\hline Abdominal Lymphadenopathy & 6 & 6.3 \\
\hline Mediastinal Lymphadenopathy & 44 & 43.1 \\
\hline Type B symptoms & 58 & 56.9 \\
\hline Visceromegaly & 24 & 25 \\
\hline \multicolumn{3}{|l|}{ B Symptoms } \\
\hline Yes & 58 & 56.3 \\
\hline No & 44 & 43.1 \\
\hline Fever & 57 & 55.9 \\
\hline Weight loss & 27 & 26.5 \\
\hline Sweating & 32 & 31 \\
\hline \multicolumn{3}{|l|}{ Histological subtype } \\
\hline Mixed cellularity & 74 & 72.5 \\
\hline Nodular sclerosis & 23 & 22.5 \\
\hline Lymphocyte depletion & 2 & 2 \\
\hline Unspecified & 3 & 3 \\
\hline \multicolumn{3}{|l|}{ Immunohistochemistry } \\
\hline CD 30 positive & 91 & 89.2 \\
\hline CD 15 positive & 50 & 49 \\
\hline \multicolumn{3}{|l|}{ Stage } \\
\hline 1 & 10 & 9.8 \\
\hline II & 33 & 32.4 \\
\hline III & 41 & 40.2 \\
\hline IV & 18 & 17.6 \\
\hline Bone marrow involvement & 8 & 7.8 \\
\hline Lungs involvement & 13 & 12.7 \\
\hline Liver involvement & 4 & 3.9 \\
\hline Spleen involvement & 24 & 23.5 \\
\hline Radiotherapy administered & 17 & 16.7 \\
\hline
\end{tabular}

\begin{tabular}{|c|c|c|}
\hline Treatment group & & \\
\hline 1 & 21 & 20.6 \\
\hline 2 & 37 & 36.3 \\
\hline 3 & 44 & 43.1 \\
\hline \multicolumn{3}{|l|}{ Outcome } \\
\hline Overall survival & 93 & 91.2 \\
\hline Event free survival & 84 & 82.4 \\
\hline
\end{tabular}

large mediastinal tumor), diagnosis of lymphocyte-predominant Hodgkin's lymphoma, post-transplant Hodgkin lymphoma and patients abandoning the treatment, were excluded from the study.

Detailed medical history, clinical examination, excision biopsy of the involved lymph node for histopathology and immunohistochemistry, echocardiography, LDH, ESR and baseline chemistry was performed for all patients. All the patients were weighed at the time of admission before start of the chemotherapy. The weight was recorded in kilograms and plotted on the standard WHO Z-score chart for age and sex. The patients were categorized as No Malnutrition, Moderate Malnutrition and Severe Malnutrition if they had $\mathrm{Z}$ score $>-2$, between $\leq-2$ to $>-3$ and $\leq-3$ respectively.

The disease was classified as per World health organization 2008 classification of HL. For staging purpose, Contrast Enhanced Computed Tomography (CECT) of neck, chest, abdomen, pelvis was done in all cases. Bulky disease was considered when the lymph node was $7 \mathrm{~cm}$ or more in size on CT, or when the mediastinal mass occupied more than one-third of the chest diameter. Because of limited availability, Positron Emission Tomography (PET) scanning was done in few cases. All patients with a stage >IIA had bilateral bone marrow aspiration and trephine biopsy. Ann Arbor staging with Cotswolds modification was used for staging purpose.

Eligible patients received chemotherapy as per EURONET-PHL protocol 2006 and were stratified into three Treatment Groups (TG). TG-1: patients of stages IA/B \& IIA without bulk and without ESR $\geq 30 \mathrm{~mm} / \mathrm{hr}$, TG-2: patients of stages IEA/B, IIEA, IIB or III A and patients of stages IA/B and IIA with bulk and/or $\geq 30 \mathrm{~mm} / \mathrm{hr}$ and TG-3: patients of stages IIEB, IIIEA/B, IIIB or IVA/B. All patients received 2 cycles of OEPA (Vincristine, Etoposide, Prednisolone, and Adriamycin) chemotherapy. Patients in TG-1 had no further chemotherapy while patients in TG-2 and TG-3 had two and four courses of COPDAC (Cyclophosphamide, Vincristine, Prednisolone, Dacarbazine) chemotherapy respectively. After two courses of OEPA chemotherapy response assessment was done with CECT scan or PET scan.

Patients with Inadequate Response (IR) received involved field radiotherapy after the end of chemotherapy. Radiotherapy dose was 19.8 Gy to all initially involved fields. Those patients with a poor response (less than $75 \%$ reduction of tumor volume in some areas or a residual mass of more than $100 \mathrm{ml}$ ) received another $10 \mathrm{~Gy}$ boost to these regions. Patients were followed up three monthly in first 2 years, four monthly in third year and six monthly in fourth and fifth year after achieving remission. Patients having relapsed or refractory disease had 2nd line chemotherapy; ABVD (Doxorubicin, Bleomycin, Vinblastine and Dacarbazine) and IEP (Ifosfamide, Etoposide and 
Table 2: Patient outcome $(n=102)$.

\begin{tabular}{|c|c|c|c|c|c|}
\hline Stage & 1 & II & III & IV & Total \\
\hline Relapse / refractory & $1(10 \%)$ & $3(9.1 \%)$ & $5(12.2 \%)$ & $1(5.6 \%)$ & $10(7.8 \%)$ \\
\hline Expired & \multirow{3}{*}{$0(0 \%)$} & $3(9.1 \%)$ & $2(5.0 \%)$ & $4(22.2 \%)$ & $9(8.8 \%)$ \\
\hline TRM & & $3(9.1 \%)$ & $2(5.0 \%)$ & $3(16.7 \%)$ & $8(7.8 \%)$ \\
\hline With disease progression & & $0(0 \%)$ & $0(0 \%)$ & $1(5.6 \%)$ & $1(1 \%)$ \\
\hline Event free survival & $9(90 \%)$ & $27(81.8 \%)$ & $34(82.9 \%)$ & $14(77.8 \%)$ & $84(82.4 \%)$ \\
\hline Overall survival & $10(100 \%)$ & $30(90.9 \%)$ & $39(95.1 \%)$ & $14(77.8 \%)$ & $93(91.2 \%)$ \\
\hline Total & 10 & 33 & 41 & 18 & 102 \\
\hline
\end{tabular}

Table 3: Comparison of current study with others studies of PHL.

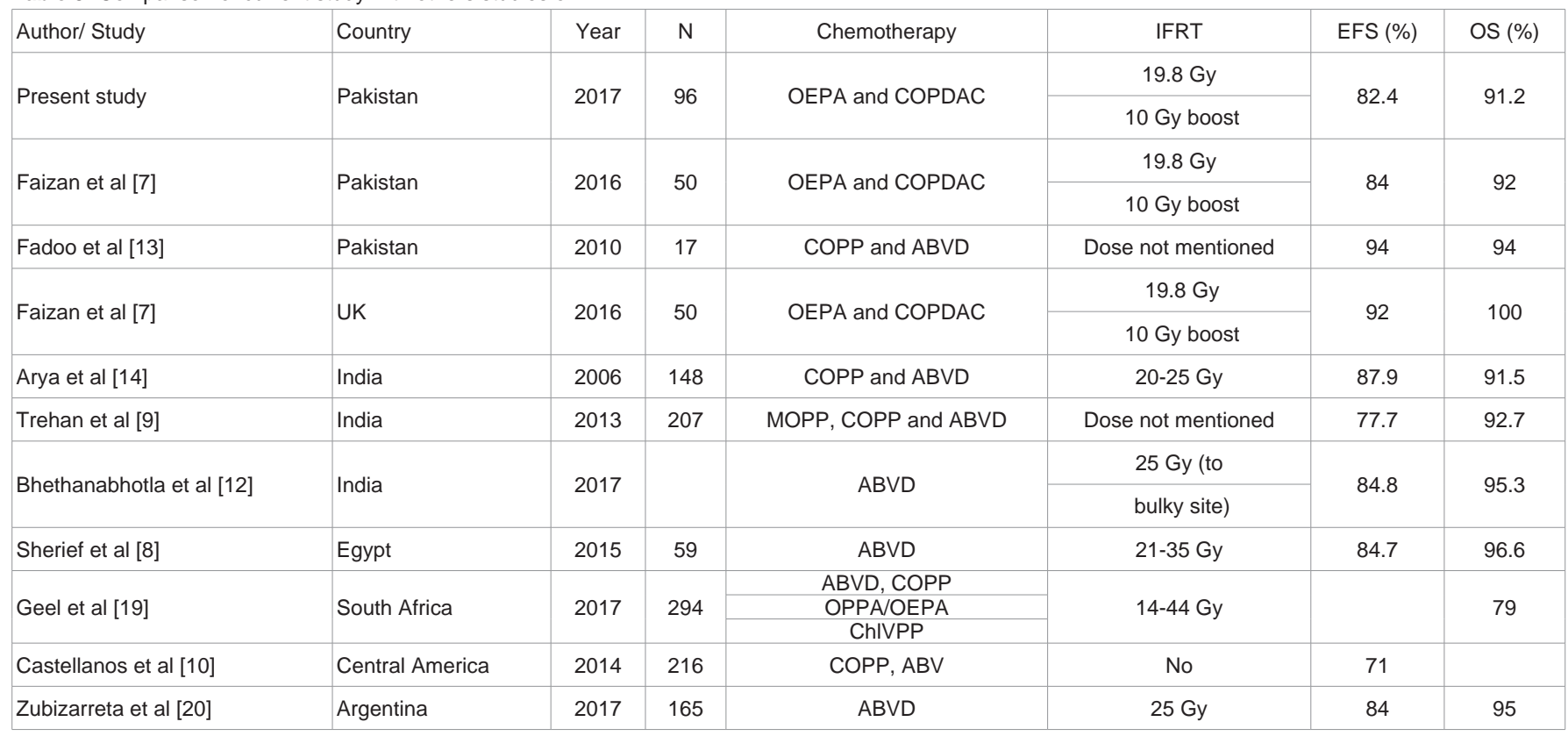

Prednisone) chemotherapy.

\section{Supportive care}

All patients were hospitalized for the initiation of chemotherapy. Subsequent chemotherapy was given as inpatient or in day care as outdoor cases. Outdoor cases were admitted immediately in case of fever or any other problem. Patients not admitted in the hospital were reviewed at least twice weekly in outdoor clinics. No prophylactic antimicrobials and colony stimulating factors were used during neutropenic period. However, all cases of febrile neutropenia were treated as inpatient with broad-spectrum intravenous antibiotics. Fever was defined as a single oral temperature of $>38^{\circ} \mathrm{C}$ or 2 readings $>37.5^{\circ} \mathrm{C}$ at least 2 hours apart. Neutropenia was defined as Absolute Neutrophil Count (ANC) of $<1000$ cells per microliter. Febrile patients with ANC $<1000$ were treated with a combination of Pipracillin-Tazobactam and Amikacin. Vancomycin or Teicoplanin were added if central venous line infection was suspected. Pipracillintazobactam was swapped with Meropenam if fever continued after 48 hours. Amphotericin B was added empirically if fever continued beyond 96hours.

Blood and blood products transfusion was given on regular basis. Haemoglobin transfusion threshold was $8.0 \mathrm{~g} / \mathrm{dL}$. Thresholds for Platelet transfusion were $10 \times 10^{9} / \mathrm{L}$ for asymptomatic patients, and
20x109/L for febrile patients.

\section{Statistical analysis}

Survival analyses were performed using the Kaplan-Meier method in SPSS 22. Chi-square test was applied and frequencies and percentages calculated. Relapse-Free Survival (RFS) was defined as the time from completion of treatment until progression of disease or relapse (RFS). Overall Survival (OS) was defined as the time from the date of diagnosis till last follow-up or death from any cause (OS). Log rank tests were used to compare survival differences. $\mathrm{P} \leq 0.05$ was considered statistically significant.

\section{Results}

During the study period, total 102 new patients of Hodgkin lymphoma were enrolled in the Paediatric oncology department at Combined Military Hospital Rawalpindi. Age at diagnosis ranged from 2.5 to 15 years and the mean and median age was $7.54( \pm$ 2.77) years and 7.0 years respectively. Majority of cases, 60 (58.8\%) were between 5 and 10 years of age. There were $80(78.4 \%)$ males and $22(21.6 \%)$ females. There were $76(74.5 \%)$ well-nourished and $26(25.5 \%)$ under-nourished children. The mean duration between symptom onset and consultation of oncologist was $8.97 \pm 8.65$ months (range from 1.0 to 48 months. Cervical lymphadenopathy was the 
most common presentation seen in 85 (83.3\%) cases. Twenty (19.6\%) patients received anti-tuberculosis treatment for variable duration before being referred to pediatric oncologist. Mixed cellularity was the most common histological subtype seen in $74(72.5 \%)$ cases followed by nodular sclerosis in $23(22.5 \%)$ cases. Immunohistochemistry demonstrated CD 30 positivity in $91(89.2 \%)$ patients and CD 15 positivity in $50(49 \%)$ patients. Demographic characteristics of the study group are summarized in (Table 1).

Ten $(9.8 \%)$ patients had stage I disease, 33 (32.4\%) had stage II, $41(40.2 \%)$ had stage III and 18 (17.6\%) had stage IV disease. Constitutional B-symptoms were present in 58 (56.9\%) patients. Bulky disease was seen in $42(41.2 \%)$ cases. Bone marrow aspiration and trephine biopsy was done in patients having $>$ II A disease. Out of $75 / 102(73.5 \%)$ patients having bone marrow examination only $8 / 102$ (7.8\%) had bone marrow involvement. Two patients had evidence of Haemophagocytic Lympho Histiocytosis (HLH) on bone marrow examination. Lung involvement was seen in $13(12.7 \%)$ patients. Liver and spleen involvement was documented in 4 (3.9\%) and 24 (23.5\%) cases. Fine Needle Aspiration Cytology (FNAC) was performed in 29 (28.4\%) cases having lymphadenopathy. However, only three cases were diagnosed as Hodgkin Lymphoma on FNAC, making only $10 \%$ specificity of this procedure.

\section{Response and Disease Outcome}

Patients were divided into three Treatment Groups (TG) as per protocol; 21 (20.6\%) patients were in TG-1, 37 (36.3\%) in TG-2 and $44(43.1 \%)$ in TG-3. Tumor response assessment was done after two cycles of OEPA chemotherapy. Eight patients (8.3\%) expired before response assessment. Out of the remaining 94 patients adequate and inadequate response was documented in 73/94 (77.6\%) and 21 (22.3\%) cases respectively. Involved Field Radiotherapy (IFRT) was administered to patients having inadequate response. However, four patients did not get IFRT and are still in remission.

Out of 10 patients having stage I disease, only one patient relapsed and was rescued with second line chemotherapy. Event Free Survival (EFS) and Overall Survival (OS) was $90 \%$ and $100 \%$ respectively in stage I disease. Out of 33 patients having stage II disease, three patients died of treatment related complications and three patients relapsed and were rescued with second line chemotherapy. EFS and OS was $81.8 \%$ and $90.9 \%$ respectively in stage II disease. Out of 41 patients having stage III disease, two patients died of treatment related complications and five patients relapsed and were rescued with second line chemotherapy. EFS and OS was $82.9 \%$ and $95.1 \%$ respectively in stage III disease. Out of 18 patients having stage IV disease, three patients died of treatment related complications and one patient relapsed. The relapsed case did not respond to second line chemotherapy and expired of progressive disease. EFS and OS was $77.8 \%$ in stage IV disease.

Out of total 102 patients in our cohort 10 patients relapsed and $9 / 10$ were rescued with 2 nd line chemotherapy. One relapsed patient required autologous stem cell transplant after ABVD \& IEP chemotherapy and one relapsed patient died of progressive disease. Eight patients (7.8\%) died of treatment related complications. Median follow-up time of our study was $1021.11 \pm 677.43$ days, ranging from 22 to 2488 days from end of treatment. At the time of analysis EFS and OS and was $84(82.4 \%)$ and $93(91.2 \%)$ respectively (Table 2).
Table 4: Results of statistical tests of association between OS and EFS and study variables in Hodgkin Lymphoma patients.

\begin{tabular}{|l|c|c|c|c|}
\hline \multirow{2}{*}{\multicolumn{1}{|c|}{ Variable }} & \multicolumn{2}{c|}{ OS } & \multicolumn{2}{c|}{ EFS } \\
\cline { 2 - 5 } & Log Rank & $P$ & Log Rank & $P$ \\
\hline Reporting time to oncologist & 17.21 & 0 & 10.46 & 0 \\
\hline Anti-tuberculosis treatment & 16.24 & 0 & 13.78 & 0 \\
\hline B Symptoms & 4.165 & 0.04 & 4.115 & 0.04 \\
\hline Bone Marrow involvement & 10.43 & 0 & 9.23 & 0 \\
\hline Lungs involvement & 10.5 & 0 & 9.162 & 0 \\
\hline Histological Subtype & 48.93 & 0 & 30.36 & 0 \\
\hline Nutritional Status & 5.726 & 0.06 & 4.001 & 0.14 \\
\hline Stage of disease & 6.114 & 0.11 & 5.758 & 0.12 \\
\hline Treatment Group (TG) & 2.449 & 0.29 & 2.283 & 0.32 \\
\hline
\end{tabular}

Febrile neutropenia was the most common complication seen in $38 / 94(40 \%)$ and $28 / 94(29.8 \%)$ cases in $1^{\text {st }}$ and $2^{\text {nd }}$ OEPA chemotherapy courses. No episode of febrile neutropenia was documented during COPDAC chemotherapy. Mucositis was seen in 12/94 (12.8\%) and $8 / 94(8.5 \%)$ cases in $1^{\text {st }}$ and $2^{\text {nd }}$ OEPA chemotherapy courses. Vincristine induced peripheral neuropathy was seen in two (2\%) cases and improved after reducing the dose of vincristine. Total 8 (7.8\%) died due to treatment related complications. Neutropenic sepsis was the main cause of death seen in 5/8 (62.5\%) cases. Treatment Related Mortality (TRM) was $17.6 \%$ in stage IV disease.

Presence of B symptoms, bone marrow involvement, duration of symptoms before start of treatment has statistically significant difference in OS and EFS of pediatric HL in the study. Age and sex were not statistically associated with outcome. (Table 3 and Figures 1-4).

\section{Discussion}

Hodgkin Lymphoma (HL) accounts for $5 \%$ to $6 \%$ of all childhood cancer [8]. There is no pediatric oncology registry in Pakistan and exact number of pediatric malignant disorders is not known. However, in the present study, HL patients were $6.8 \%$ of total new cases making it in accordance with the international data.

There is substantial variation in epidemiological, clinical, and pathological features of PHL, according to various geographic areas. Patients in developing countries tend to present at a younger age as compared with the western world where the mean age of presentation is between 12 and 15 years $[4,9,10]$. In the present study, the mean age of diagnosis was 7.5 years and the majority of cases $(58.8 \%)$ were between 5 and 10 years of age. Other studies report similar age of diagnosis from developing countries in Asia and Africa, indicating the association of HL with Epstein-Barr Virus (EBV) infection in these countries [4,7-11]. Many authors have reported male predominance in HL $[4,6,8,12]$. In our study, male to female ratio was 3:6:1. Similar male to female ratio is reported from another study from Pakistan [7]. The reason for this male predominance is not clearly understood. One reason may be gender discrimination still prevalent in many developing countries.

Patients were referred to pediatric oncologist very late. The mean reporting time to pediatric oncologist was $8.97 \pm 8.65$ months. This 
delayed presentation was associated with statistically significant difference in EFS and OS. This can be due to the fact that delayed presentation leads to more advanced stage. Because tuberculosis is very common in Pakistan and children presenting with fever, weight loss and cervical lymphadenopathy are suspected to have tuberculosis. In the present study, cervical lymphadenopathy was seen in $85(83.3 \%)$ cases and B symptoms were present in 58 (56.3\%) cases. Twenty (19.6\%) patients received anti-tuberculosis treatment for variable duration before reporting to paediatric oncologist. Faizan et al [7] have also documented use of anti-tuberculosis treatment for several weeks before initiating investigations for lymphoma.

In the present study, the most common histological subtype was mixed cellularity. Faizan et al, [7] reported that $64 \%$ children had MC in their study. Other studies from developing countries have reported similar results $[4,7-9,13,14]$. The earlier presentation of MC subtype of HL in young males in low-income countries is associated with high prevalence of EBV infection, overcrowding and malnutrition $[4,6,7,13]$.

In the present study, majority of patients (about 58\%) had advanced stage (Stage III and IV) disease. Similar pattern of advanced disease is reported by other authors from low-income countries, most likely due to delay in diagnosis and referral to oncologists $[7-9,14]$. Contrary to this, in the western world, most of the newly diagnosed patients have early stage (stage I-II) disease [8].

Over the last three decades, treatment protocols for PHL have been modified with an aim to reduce radiotherapy and modify chemotherapy to achieve high cure rates with minimal toxicity and long-term side effects [1,15-18]. There is no uniform treatment protocol for $\mathrm{HL}$ in Pakistan and other developing countries. In the present study, EURONET-PHL protocol 2006 was used for treatment. The patients were stratified into three Treatment Groups (TG). The patients in TG 1 and 2 having low stage disease showed better overall survival as compare to children with advanced disease. In the present study, the 5-years OS and EFS was $91.2 \%$ and $82.4 \%$ respectively. Faizan et al [7] from Pakistan used same treatment protocol and reported very similar 5-years OS and EFS as $92 \%$ and $84 \%$ respectively. Other studies from developing countries reported similar results [7-10,12-14,19, 20]. (Summarized in Table 3).

In the present study, eight patients died, resulting in Treatment Related Mortality (TRM) of 7.8\%. Neutropenic sepsis was the leading cause of death in five cases. Other causes included respiratory failure due to bilateral lung involvement in one case, hepatic failure due to disease involvement in one case and sudden death at home in one case. Presence of B symptoms, delayed presentation to pediatric oncologist, bone marrow involvement at the time of diagnosis and histological subtype had statistically significant impact on overall survival. Nutritional status also had impact on survival though it was not statistically significant. Survival was $94.7 \%$ in children having normal nutritional status and it decreased to $75 \%$ in children suffering from severe malnutrition (log rank $\mathrm{p}=0.057$ ). Other studies have also documented increased TRM and inferior EFS in malnourished children $[6,21,22]$. Delay in start of treatment for HL also had statistically significant impact on OS and EFS. OS decreased from $95.5 \%$ to $61.5 \%$ (log rank $\mathrm{p}=0.000$ ) in children presenting before and after one year from onset of symptoms. A delay in diagnosis especially in the presence of B symptoms results in worsening of the nutritional status of the children due to decreased caloric intake because of loss of appetite and increased basal metabolic rate secondary to fever and infection. Delay in diagnosis also leads to bone marrow involvement, which is also a bad prognostic factor. Please see (Table 4) for Results of Statistical Tests of Association between OS and EFS and Study Variables.

\section{Conclusion}

The presentstudy has documented that young males having cervical lymphadenopathy with B symptoms is the common presentation of PHL in Pakistan. Mixed cellularity is the most common subtype of PHL. Long duration of disease before presentation, malnutrition, presence of B symptoms, bone marrow and lungs involvement and advanced disease is associated with decreased OS and EFS.

\section{Acknowledgement}

All authors contributed to writing and editing the paper. We are thankful to Mr Adnan Ghafoor for his contribution as data manager of our cases.

\section{References}

1. Dorffel W, Luders $\mathrm{H}$, Ruhl $U$, Albrecht M, Marciniak $\mathrm{H}$, Parwaresch R, et al. Preliminary results of the multicenter trial $\mathrm{GPOH}-\mathrm{HD} 95$ for the treatment of Hodgkin's disease in children and adolescents: analysis and outlook. Klinische Padiatrie. 2003; 215: 139-45.

2. Board PDQPTE. Childhood Hodgkin Lymphoma Treatment (PDQ(R)): Health Professional Version. PDQ Cancer Information Summaries. Bethesda (MD): National Cancer Institute (US). 2002.

3. Smith MA, Altekruse SF, Adamson PC, Reaman GH, Seibel NL. Declining childhood and adolescent cancer mortality. Cancer. 2014; 120: 2497-2506.

4. Ganesan P, Kumar L, Raina V, Sharma A, Bakhshi S, Sreenivas V, et al. Hodgkin's lymphoma--long-term outcome: an experience from a tertiary care cancer center in North India. Annals of hematology. 2011; 90: 1153-1160.

5. White Y, Castle VP, Haig A. Pediatric oncology in developing countries: challenges and solutions. The Journal of pediatrics. 2013; 162: 1090-1091.

6. Molyneux E, Scanlan T, Chagaluka G, Renner L. Haematological cancers in African children: progress and challenges. Br J Haematol. 2017; 177: 971978.

7. Faizan M, Taj MM, Anwar S, Asghar N, Ahmad A, Lancaster D, et al. Comparison of Presentation and Outcome in 100 Pediatric Hodgkin Lymphoma Patients Treated at Children Hospital, Lahore, Pakistan and Royal Marsden Hospital, UK. Journal of the College of Physicians and Surgeons-Pakistan: JCPSP. 2016; 26: 904-7.

8. Sherief LM, Elsafy UR, Abdelkhalek ER, Kamal NM, Elbehedy R, Hassan $\mathrm{TH}$, et al. Hodgkin lymphoma in childhood: clinicopathological features and therapy outcome at 2 centers from a developing country. Medicine. 2015; 94: e670.

9. Trehan A, Singla S, Marwaha RK, Bansal D, Srinivasan R. Hodgkin lymphoma in children: experience in a tertiary care centre in India. Journal of pediatric hematology/oncology. 2013; 35: 174-179.

10. Castellanos EM, Barrantes JC, Baez LF, Gamboa Y, Pena A, Alabi S, et al. A chemotherapy only therapeutic approach to pediatric Hodgkin lymphoma: AHOPCA LH 1999. Pediatr Blood Cancer. 2014; 61: 997-1002.

11. Dinand V, Arya LS. Epidemiology of childhood Hodgkins disease: is it different in developing countries? Indian pediatrics. 2006; 43: 141-147.

12. Bhethanabhotla S, Jain S, Kapoor G, Mahajan A, Chopra A, Vishnubhatla $S$, et al. Outcome of pediatric advanced Hodgkin lymphoma treated with ABVD and predictors of inferior survival: a multicenter study of 186 patients. Leukemia \& lymphoma. 2017; 58: 1617-1623. 
13. Fadoo Z, Belgaumi A, Alam M, Azam I, Naqvi A. Pediatric lymphoma: a 10 year experience at a tertiary care hospital in Pakistan. Journal of pediatric hematology/oncology. 2010; 32: e14-e18.

14. Arya LS, Dinand V, Thavaraj V, Bakhshi S, Dawar R, Rath GK, et al. Hodgkin's disease in Indian children: outcome with chemotherapy alone. Pediatr Blood Cancer. 2006; 46: 26-34.

15. Mauz-Korholz C, Hasenclever D, Dorffel W, Ruschke K, Pelz T, Voigt A, et al. Procarbazine-free OEPA-COPDAC chemotherapy in boys and standard OPPA-COPP in girls have comparable effectiveness in pediatric Hodgkin's Iymphoma: the GPOH-HD-2002 study. Journal of clinical oncology: official journal of the American Society of Clinical Oncology. 2010; 28: 3680-3686.

16. Ruhl U, Albrecht M, Dieckmann K, Luders H, Marciniak H, Schellenberg D, et al. Response-adapted radiotherapy in the treatment of pediatric Hodgkin's disease: an interim report at 5 years of the German GPOH-HD 95 trial. International journal of radiation oncology, biology, physics. 2001; 51: 120912018.

17. Schellong G. Treatment of children and adolescents with Hodgkin's disease the experience of the German-Austrian Paediatric Study Group. Bailliere's clinical haematology. 1996; 9: 619-634.

18. Schellong G, Potter R, Bramswig J, Wagner W, Prott FJ, Dorffel W, et al High cure rates and reduced long-term toxicity in pediatric Hodgkin's disease: the German-Austrian multicenter trial DAL-HD-90. The German-Austrian Pediatric Hodgkin's Disease Study Group. Journal of clinical oncology: officia journal of the American Society of Clinical Oncology. 1999; 17: 3736-3744.

19. Geel JA, Chirwa TC, Rowe B, Eyal KC, Omar F, Stones DK, et al. Treatment outcomes of children with Hodgkin lymphoma between 2000 and 2010: First report by the South African Children's Cancer Study Group. Pediatr Blood Cancer. 2017; 64.

20. Zubizarreta PA, Alfaro E, Guitter M, Sanchez La Rosa C, Galluzzo ML, Millan $\mathrm{N}$, et al. Children and Adolescent Hodgkin Lymphoma in Argentina: Longterm Results After Combined ABVD and Restricted Radiotherapy. Journal of pediatric hematology/oncology. 2017; 39: 602-608.

21. Park S, Han B, Cho JW, Woo SY, Kim S, Kim SJ, et al. Effect of nutritional status on survival outcome of diffuse large B-cell lymphoma patients treated with rituximab-CHOP. Nutrition and cancer. 2014; 66: 225-233.

22. Pribnow AK, Ortiz R, Baez LF, Mendieta L, Luna-Fineman S. Effects of malnutrition on treatment-related morbidity and survival of children with cancer in Nicaragua. Pediatr Blood Cancer. 2017; 64
Ann Hematol Oncol - Volume 5 Issue 6 - 2018

ISSN : 2375-7965 | www.austinpublishing group.com

Ghafoor et al. () All rights are reserved
Citation: Ghafoor T, Iftikhar, Sharif I, Ashraf T, Ahmed S, Bashir F. Pediatric Hodgkin Lymphoma; Experience with Euronet Pediatric Hodgkin Lymphoma Protocol in a Developing Country. Ann Hematol Oncol. 2018; 5(6): 